\title{
Improving $\mathrm{CoQ}_{10}$ productivity by strengthening glucose transmembrane of Rhodobacter sphaeroides
}

Yuying Yang ${ }^{1,2 \dagger}, \mathrm{Lu} \mathrm{Li}^{1,2+}$, Haoyu Sun ${ }^{1,2}$, Zhen $\mathrm{Li}^{1,2}$, Zhengliang Qi ${ }^{* *}$ and Xinli Liu ${ }^{1,2^{*}}$

\begin{abstract}
Background: Several Rhodobacter sphaeroides have been widely applied in commercial $\mathrm{CoQ}_{10}$ production, but they have poor glucose use. Strategies for enhancing glucose use have been widely exploited in $R$. sphaeroides. Nevertheless, little research has focused on the role of glucose transmembrane in the improvement of production.

Results: There are two potential glucose transmembrane pathways in $R$. sphaeroides ATCC 17023: the fructose specific-phosphotransferase system (PTS Fru, fruAB) and non-PTS that relied on glucokinase ( $g / k)$. fruAB mutation revealed two effects on bacterial growth: inhibition at the early cultivation phase (12-24 h) and promotion since $36 \mathrm{~h}$. Glucose metabolism showed a corresponding change in characteristic vs. the growth. For $\triangle$ fruA $\triangle$ fruB, maximum biomass $\left(\mathrm{Bio}_{\max }\right)$ was increased by $44.39 \%$ and the $\mathrm{CoQ}_{10}$ content was $27.08 \%$ more than that of the WT. glk mutation caused a significant decrease in growth and glucose metabolism. Over-expressing a galactose: $\mathrm{H}^{+}$symporter (galP) in the $\triangle$ fruA $\triangle$ fruB relieved the inhibition and enhanced the growth further. Finally, a mutant with rapid growth and high $\mathrm{CoQ}_{10}$ titer was constructed ( $\triangle$ fruA $\triangle$ fruB/tac::galP $\mathrm{OP}_{\mathrm{P}}$ ) using several glucose metabolism modifications and was verified by fermentation in $1 \mathrm{~L}$ fermenters.
\end{abstract}

Conclusions: The PTS Fru mutation revealed two effects on bacterial growth: inhibition at the early cultivation phase and promotion later. Additionally, biomass yield to glucose $\left(Y_{b / g l c}\right)$ and $\mathrm{CoQ}_{10}$ synthesis can be promoted using fruAB mutation, and $g / k$ plays a key role in glucose metabolism. Strengthening glucose transmembrane via non-PTS improves the productivity of $\mathrm{CoQ}_{10}$ fermentation.

Keywords: R. sphaeroides, Glucose transmembrane, Glucose metabolism, $\mathrm{CoQ}_{10}$ productivity

\section{Introduction}

Glucose is a common monosaccharide that is available in abundance. As glucose is cheap and easy to use for microorganisms, it can serve as an ideal source of carbon for producing high-value products, such as $\mathrm{CoQ}_{10}$, through

\footnotetext{
*Correspondence: qizhengliang@qlu.edu.cn; vip.Ix|@163.com

†Yuying Yang and Lu Li have equal contribution to the study

1 Key Laboratory of Shandong Microbial Engineering, College

of Bioengineering, Qilu University of Technology (Shandong Academy of Sciences), Jinan, Shandong, People's Republic of China

${ }^{2}$ State Key Laboratory of Bio-Based Material and Green Papermaking (LBMP), Qilu University of Technology (Shandong Academy of Sciences),

Jinan, Shandong, People's Republic of China
}

microbial fermentation [1-3]. Studies related to microbial glucose metabolism have always been a hot topic in industrial microbiology [1, 4]. As glucose metabolism pathways have been well established for many microorganisms, many novel biotechnologies, particularly metabolic engineering, synthetic biology, and systems biology, have been applied to modify intracellular metabolic pathways to enhance the glucose utilization efficiency in microorganisms $[1,4,5]$. Besides the functional enzymes for glucose metabolism, glucose utilization also requires a set of genes that encode specific transporters and regulators [5]. Glucose transmembrane is an important step because exogenous glucose cannot go into cells through 
free diffusion and must rely on a transporter to cross the cell membrane [6]. Recently, researchers have realized the importance of glucose transport efficiency during microbial fermentation and focus on microbial sugar transmembrane studies [6-8]. So far, the sugar transmembrane mechanisms of many industrial microbes are still unknown, limiting metabolic modification of sugar transmembrane in these microbes.

Microorganisms depend on more than one system to transport exogenous glucose; the glucose transmembrane mechanisms for $E$. coli have been widely investigated [6, 7]. E. coli can use two pathways for glucose transmembrane: phosphoenolpyruvate (PEP): carbohydrate phosphotransferase system (PTS) and non-PTS [6]. The non-PTS include the ATP binding cassette (ABC) system and the major facilitator superfamily (MFS) system. PTS ${ }^{\text {Glc }}$ is a multiprotein phosphorelay system that accompanies the import and simultaneous phosphorylation of carbohydrates. Since the discovery of the PTS ${ }^{\text {Glc }}$ in E. coli, it has existed in many other bacteria [9]. It has been confirmed that the PTS ${ }^{\text {Glc }}$ is primarily composed of enzymes including $\mathrm{IICB}^{\mathrm{Glc}} / \mathrm{IIA}^{\mathrm{Glc}}$ (EIIs), $\mathrm{HPr}$, and enzyme $\mathrm{I}$ (EI). EI and HPr, the two sugar-nonspecific protein constituents of the PTS, are soluble cytoplasmic proteins participating in the transport of all PTS carbohydrates [6]. EIIs are sugar-specific transporters connecting the common PEP/EI/HPr phosphoryl transfer pathway. PTS ${ }^{\text {Glc }}$ is considered an effective way to use glucose because only one phosphoenolpyruvate is coupled with the translocation phosphorylation of glucose when forming an ATP. In contrast, use of glucose through the $\mathrm{ABC}$ transporter requires extra ATP for glucose phosphorylation in the carbohydrate kinase reaction $[6,10]$. Therefore, PTS ${ }^{\mathrm{Glc}}$ is a preferred channel for transferring exogenous glucose into cells in industrial bacteria, such as E. coli, Bacillus subtilis, and Corynebacterium glutamicum [4, 11, 12]. $E$. coli activates the non-PTS system for glucose transmembrane when exogenous glucose concentration is low $(<1 \mathrm{mM})$, or PTS ${ }^{\mathrm{Glc}}$ function is defective [7]. Some bacteria lacking PTS ${ }^{\mathrm{Glc}}$, such as Pseudomonas putida, utilize the $\mathrm{ABC}$ system to transfer exogenous glucose into cells [9].

$R$. sphaeroides has received significant attention because of its wide biotechnological applications, such as its ability to synthesize a high content of $\mathrm{CoQ}_{10}$, carotenoids, and isoprenoids as a source of pharmaceutical materials [13-16]. $\mathrm{CoQ}_{10}$ is an oil-soluble quinone that has a decaprenyl side chain. So far, it has been widely used in functional food and cosmetics industries because of its antioxidant function. Researchers have recently found that $\mathrm{CoQ}_{10}$ can regulate several genes that play an important role in cholesterol metabolism, inflammatory responses, or both [14]. Moreover, it is beneficial to patients with cardiovascular diseases, hypertension, and Parkinson's disease [17]. Compared with the methods of animal and plant extraction and chemosynthesis, the production of $\mathrm{CoQ}_{10}$ by microbial fermentation is lowcost, safe, and efficient [16]. Additionally, $R$. sphaeroides, a $C o Q_{10}$ producer with high contents of $C_{0} Q_{10}$, has been widely used in the industrial production of $\mathrm{CoQ}_{10}$. For the commercial production of $\mathrm{CoQ}_{10}$ with microbial fermentation, glucose acts as a major carbon source. Various strategies for guiding the metabolic flux toward $\mathrm{CoQ}_{10}$ biosynthesis have been exploited for $R$. sphaeroides [16]. Nevertheless, presently, there is little research on improving $\mathrm{CoQ}_{10}$ production by modifying the glucose transmembrane. Although $R$. sphaeroides has a wide spectrum of carbon source utilization, it had a low glucose consumption rate than E. coli $[18,19]$. Fuhrer et al. reported that the glucose uptake rate of $R$. sphaeroides was $1.8 \pm 0.1 \mathrm{mmol} / \mathrm{g}$ dry cells weight $(\mathrm{DCW}) / \mathrm{h}$, which was only approximately $23.07 \%$ as that of E. coil [19]. Therefore, the glucose transmembrane process of $R$. sphaeroides was a bottle-neck step for glucose metabolism, which may be a new way to further promote the productivity of $\mathrm{CoQ}_{10}$.

Considering the importance of glucose transmembrane for glucose metabolism and poor glucose utilization efficiency of $R$. sphaeroides, the first potential pathway of glucose transmembrane of $R$. sphaeroides were analyzed in this work. Later, a deep study was conducted to show the function of these pathways on glucose metabolism. Finally, we evaluated the influence of glucose transmembrane on $\mathrm{CoQ}_{10}$ synthesis efficiency and optimized $\mathrm{CoQ}_{10}$ fermentation by $R$. sphaeroides by modifying glucose transmembrane. Moreover, $R$. sphaeroides ATCC 17023 is a paradigmatic organism among isolated $R$. sphaeroides strains with clear genetic background and mature genetic manipulation tools that we chose as a research object in this study [20].

\section{Material and methods \\ Strains, media and growth conditions}

All the strains used in this study were summarized in Table 1. R. sphaeroides ATCC 17023 (wild type, WT) was used as the parental strain in this work. The mutant strains were constructed in this background. E. coli JM109 was used as a plasmid host, and E. coli S17-1 was used to conjugate DNA into $R$. sphaeroides. $R$. sphaeroides ATCC 17023 and mutant strains were routinely cultivated at $32^{\circ} \mathrm{C}$ in medium A $(3 \mathrm{~g} / \mathrm{L}$ glucose, $2 \mathrm{~g} / \mathrm{L}$ $\mathrm{NaCl}, 8 \mathrm{~g} / \mathrm{L}$ yeast extract, $0.256 \mathrm{~g} / \mathrm{L} \mathrm{MgSO}_{4} \cdot 7 \mathrm{H}_{2} \mathrm{O}, 1.3 \mathrm{~g} / \mathrm{L}$ $\mathrm{KH}_{2} \mathrm{PO}_{4}, 15 \mu \mathrm{g} / \mathrm{L}$ biotin, $1 \mathrm{mg} / \mathrm{L}$ thiamine hydrochloride, $1 \mathrm{mg} / \mathrm{L}$ nicotinic acid, $\mathrm{pH}$ 7.2) as seed (exponentially phase cells, $\mathrm{OD}_{600} \geq 2$ ). R. sphaeroides cultures were incubated at $32^{\circ} \mathrm{C}$ in modified Sistrom's minimal medium 
Table 1 Strains and plasmids used in this work

\begin{tabular}{|c|c|c|}
\hline Strains and plasmids & Description & Reference or source \\
\hline \multicolumn{3}{|l|}{ Strains } \\
\hline Wild-type & Rhodobacter sphaeroides ATCC 17025 & Lab preservation \\
\hline$\Delta g / k$ & glk markerless deletion mutant & This work \\
\hline$\triangle$ fruA $\triangle$ fruB & fruAB markerless deletion mutant & This work \\
\hline$\triangle$ fruA $\triangle$ fruB/bp & $\triangle$ fruA $\triangle$ fruB harboring pBBR1MCS-2 & This work \\
\hline$\triangle$ fruA $\triangle$ fruB/galP $P_{O P}$ & $\triangle$ fruA $\triangle$ fruB harboring pBBR1MCS-2:: galP & This work \\
\hline$\triangle$ fruA $\triangle$ fruB/tac::galP $O P$ & $\triangle$ fruA $\triangle$ fruB harboring pBBR1MCS-2:: tac:: galP & This work \\
\hline$\triangle$ fruA $\triangle$ fruB/tac::glk $O P$ & $\triangle$ fruA $\triangle$ fruB harboring pBBR1MCS-2:: tac::glk & This work \\
\hline E. colis17-1 & recA, harboring the genes tra, proA, thi-1 (pRP4-2-Tc::Mu-Km::Tn7) & Lab preservation \\
\hline \multicolumn{3}{|l|}{ Plasmids } \\
\hline pK18mobsacB & suicide vector, sacB (sucrose sensitivity), $\mathrm{Km}^{\mathrm{r}}$ & Lab preservation \\
\hline pBBR1MCS-2 & ori pBBR1, lacZa, $\mathrm{Km}^{r}$, used for geng over-expression & Lab preservation \\
\hline pBBR1MCS-2::tac & pBBR1MCS-2 harboring the tac promoter & Lab preservation \\
\hline pK18mobsacB::glk-L-R & For glk deletion & This work \\
\hline pK18mobsacB::fruA-L-R & For fruA deletion & This work \\
\hline pK18mobsacB::fruB-L-R & For fruB deletion & This work \\
\hline pBBR1MCS-2::galP & For galP expression & This work \\
\hline pBBR1MCS-2::tac::galP & For galP expression with strong promoter tac & This work \\
\hline pBBR1MCS-2::tac::glk & For glk expression with strong promoter tac & This work \\
\hline
\end{tabular}

(MSMM) lacking succinate and with glucose as an alternative carbon source $(6.5 \mathrm{~g} / \mathrm{L})$ [18]. Before inoculation the seeds of these strains were washed with sterilized PBS ( $\mathrm{pH}$ 7.2), and then resuspended with the same solution to adjust the cell density equal $\left(\mathrm{OD}_{600} 1\right)$. Antibiotics were added into the medium A and MSMM when necessary. E. coli JM109 and E. coli S17-1 were grown at $37^{\circ} \mathrm{C}$ in Luria-Bertani (LB) medium with antibiotics when necessary. The concentrations of antibiotics and chemicals used in the construction of plasmids and recombinant strains were as follows: $25 \mu \mathrm{g} / \mathrm{mL}$ kanamycin and $150 \mu \mathrm{g} / \mathrm{mL} \mathrm{K}_{2} \mathrm{TeO}_{3}$ for $R$. sphaeroides, and $100 \mu \mathrm{g} / \mathrm{mL}$ kanamycin for $E$. coli strains. Lab-scale fermentation of $\mathrm{CoQ}_{10}$ was carried out with medium B containing $40 \mathrm{~g} / \mathrm{L}$ glucose, $6.3 \mathrm{~g} / \mathrm{L} \mathrm{MgSO} 4,4 \mathrm{~g} / \mathrm{L}$ corn steep liquor, $3 \mathrm{~g} / \mathrm{L}$ sodium glutamate, $3 \mathrm{~g} / \mathrm{L}(\mathrm{NH} 4)_{2} \mathrm{SO}_{4}, 3 \mathrm{~g} / \mathrm{L} \mathrm{KH}_{2} \mathrm{PO}_{4}$, $2 \mathrm{~g} / \mathrm{L} \mathrm{CaCO}_{3}, 1 \mathrm{mg} / \mathrm{L}$ nicotinic acid, $1 \mathrm{mg} / \mathrm{L}$ thiamine hydrochloride, and $15 \mu \mathrm{g} / \mathrm{L}$ biotin, supplemented with $25 \mu \mathrm{g} / \mathrm{mL}$ kanamycin.

\section{Plasmids construction}

Plasmids used in this study were listed in Table 1, and the related primers and restriction enzymes were presented in Additional file 6: Table S1. pK18mobsacB::glkL-R was constructed with splicing by overlap extension (SOE) PCR. Briefly, an upstream and downstream fragment of $g l k$ was amplified with primer pair $g l k$-L-F/glk$\mathrm{L}-\mathrm{R}$ and $g l k-\mathrm{R}-\mathrm{F} / g l k-\mathrm{R}-\mathrm{R}$ using $R$. sphaeroides ATCC 17025 genomic DNA as template, respectively. The two fragments were joined by SOE PCR to generate a 1,560-bp fragment, which was digested and ligated into the PstI/SphI sites of pK18mobsacB to obtain pK18mobsacB::glk-L-R. The details of experiment were presented in Additional file 1: Fig. S1. An upstream and downstream fragment of $f r u A$ was amplified with primer pair fruA-L-F/fruA-L-R and fruA-R-F/fruA $-\mathrm{R}-\mathrm{R}$. The two fragments were joined by SOE PCR to generate a 1,819-bp fragment, which was digested and ligated into the SalI/EcoRI sites of pK18mobsacB to obtain pK18mobsacB::furA-L-R. The construction of pK18mobsacB::fruB-L-R obeyed to the same procedure of pK18mobsacB::furA-L-R construction. The two fragments were amplified with primer pair fruB-L-F/fruB$\mathrm{L}-\mathrm{R}$ and $f r u B-\mathrm{R}-\mathrm{F} /$ fruA-R-R, and then were joined by SOE PCR to generate a 1399-bp fragment. Afterwards, the fragment was ligated into the EcoRI/SphI sites of pK18mobsacB to obtain pK18mobsacB::furB-L-R. The details of the two plasmids construction were shown in Additional file 2: Fig. S2. The wide host-range conjugative plasmids pBBR1MCS-2 and pBBR1MCS-2::tac were used to construct the over-expression vectors. For the construction of pBBR1MCS-2::tac:: galP, the entire open reading frame of galP gene was amplified by PCR with primer pair galP $_{o p}-\mathrm{F} /$ galP ${ }_{o p}-\mathrm{R}$ using E. coil K-12 substr. MG1655 genomic DNA as template. After digestion with $X b a \mathrm{I} / S a c \mathrm{I}$, it was ligated into pBBR1MCS-2::tac to obtain pBBR1MCS-2::tac::galP. The details of pBBR1MCS2::tac::galP construction were presented in Additional 
file 3: Fig. S3. Simultaneously, pBBR1MCS-2::galP was obtained following the same method of pBBR1MCS2::tac::galP construction.

\section{Strains construction}

$\Delta g l k$ and $\triangle f r u A \Delta f r u B$ were constructed as in-frame markerless deletion of almost the entire open reading frames by a two-step recombination strategy, which was based on diparental conjugation as previously described [14]. In short, E. coli S-17 bearing the target plasmid was used as the donor strain, and $R$. sphaeroides ATCC 17023 was used as the recipient strain. The donor/ recipient ratio was $1: 7$ for conjugation and the colonies resistant to $150 \mu \mathrm{g} / \mathrm{mL} \mathrm{K}_{2} \mathrm{TeO}_{3}$ and $25 \mu \mathrm{g} / \mathrm{mL}$ kanamycin were picked and cultivated for plasmid extraction and sequencing verification. For the construction of $\Delta g l k$, the pK18mobsacB::glk-L-R was transformed into $R$. sphaeroides ATCC 17023 by diparental conjugation. Clones obtained from single homologous recombination event were selected on kanamycin $/ \mathrm{K}_{2} \mathrm{TeO}_{3}$ supplemented medium A agar plates, and then grown for 48-72 $\mathrm{h}$ in medium without antibiotic, followed by serial dilution and plating onto SMM plates containing $10 \%(\mathrm{w} / \mathrm{w})$ sucrose to select for a second crossover event. Finally, sucrose-resistant and kanamycin-sensitive colonies were selected as potential positive transformants and verified by PCR using $g l k$-P1/glk-P4 primers. To obtain the mutant $\triangle$ fruA $\triangle$ fruB, $\triangle$ fruA was firstly constructed, and then the gene fruB was knocked out of the genome of $\triangle f r u A$ further. The method was similar to that of the construction of $\Delta g l k$. The $\triangle f r u A \Delta f r u B$ was verified by primer pair fruA-P1/fruA-P4 and fruB-P1/fruB-P4. For the construction of $\triangle$ fruA $\triangle$ fruB/tac::galP ${ }_{O P}$, pBBR1MCS2::tac::galP was transformed into $\triangle$ fruA $\triangle$ fruB by diparental conjugation, and selected on medium A supplemented with kanamycin $(25 \mu \mathrm{g} / \mathrm{mL})$ and $\mathrm{K}_{2} \mathrm{TeO}_{3}(150 \mu \mathrm{g} / \mathrm{mL})$. Afterwards, the resistant clones were selected and verified. Also, other gene over-expression mutants were constructed following the same method. Primers used for mutant strains verification were listed in Additional file 7: Table S2.

\section{RNA extraction and RT-qPCR assay}

Different growth-phase cells $\left(1 \times 10^{7}\right)$ cultured in MSMM medium were harvested by centrifugation at $8000 \times g$ for $3 \mathrm{~min}$ at $4{ }^{\circ} \mathrm{C}$. Total RNA was extracted from R. sphaeroides using a Total RNA Extraction Kit and purified as described by the procedures. Synthesis of cDNA was performed by the reverse transcription reaction according to the HiFiScript cDNA Synthesis Kit instructions. Cham Q Universal SYBR qPCR master mix (Vazyme, Nanjing, China; Q711-02) and the ABI First Step Quantitative Polymerase Chain Reaction System
(Applied Biosystems, San Mateo, CA, USA) were used to perform quantitative polymerase chain reactions under the following reaction conditions: $95{ }^{\circ} \mathrm{C}$ for $3 \mathrm{~min}$, followed by 40 cycles of $95{ }^{\circ} \mathrm{C}$ for $10 \mathrm{~s}, 56{ }^{\circ} \mathrm{C}$ for $30 \mathrm{~s}$, and $72{ }^{\circ} \mathrm{C}$ for $30 \mathrm{~s}$. As an internal standard control, the relative abundance of $16 \mathrm{~S}$ rRNA was used to standardize the results. All assays were performed in triplicate. Primers used in RT-qPCR were listed in Additional file 8: Table S3. The result of RT-qPCR was calculated based on the following formula:

$$
2^{-\Delta \Delta \mathrm{Ct}}=2^{-[(\mathrm{Ct} \text { of Gi/A-Ct of Gc/A })-(\mathrm{Ct} \text { of Gi/B-Ct of Gc/B })\}},
$$

where $G_{i / A}$ is the gene of interest in sample $A, G_{c / A}$ is the gene of internal control in sample $A, G_{i / B}$ is the gene of interest in sample $B$ and $G_{c / B}$ gene of internal control in sample B.

\section{$\mathrm{CoQ}_{10}$ fermentation in Lab-scale bioreactors}

For the lab-scale fermentation of $\mathrm{CoQ}_{10}$, three 1-L quadruple fermentation tanks (Bench-Top Bioreactor Multifors 2 type, INFORS HT, Switzerland) were used. The operation procedure was based on the method of Zhang et al. [13]. Seeds of $R$. sphaeroides were prepared as above shaking-flask cultivation. Subsequently, $16 \mathrm{~mL}$ of seeds were inoculated into tank with $0.8 \mathrm{~L}$ sterilizing medium B supplemented with $25 \mu \mathrm{g} / \mathrm{mL}$ kanamycin, and then, cultured at $32{ }^{\circ} \mathrm{C}$ for $96 \mathrm{~h}$. The $\mathrm{pH}$ was maintained at 7.0. The aeration and agitation protocol were $1 \mathrm{vvm}$ and $400 \mathrm{rpm}$ during the whole fermentation. Bacterial growth $\left(\mathrm{OD}_{600}\right)$, glucose concentration and $\mathrm{CoQ}_{10}$ titer were detected each $12 \mathrm{~h}$.

\section{Analytical methods}

Cultured broth was fetched each $12 \mathrm{~h}$ for biomass determination at $\mathrm{OD}_{600}$ by spectrophotometer (MAPADA INSTRUMENTSUV-1800, China) and calculated using a calibration curve which indicated the relationship between $\mathrm{OD}_{600}$ and dry cell weight (DCW) $\left(1 \mathrm{DD}_{600}\right.$ approximately equaled to $0.40 \mathrm{~g} \mathrm{DCW} / \mathrm{L})$. In this study, $100 \mathrm{~mL}$ of cultivated broth were centrifugated to obtain cell pellets, and then used to get dry cells through lyopilization for DCW measuring. Residual glucose was detected by a SBA-40 Biosensor (Biology Institute of Shandong Academy of Sciences, China). pH was measured at the starting and end of cultivation by a SevenCompact $^{\mathrm{TM}} \mathrm{pH}$ meter S220 (METTLER TOLEDO, China). The method of extraction and quantification of $\mathrm{CoQ}_{10}$ was according to Zhang et al. [13]. In the study, all experiments were repeated three times. The data shown in the corresponding tables and figures were the mean values of the experiments and the error bars indicated the standard deviation. Data was treated via one-way 
ANOVA method $(\mathrm{P}<0.05)$.Statistical significance was determined using the SAS statistical analysis program, version 8.01 (SAS Institute, Cary, NC, USA).

\section{Results}

\section{Potential glucose transmembrane pathways for $R$.} sphaeroides ATCC 17023

By retrieving the NCBI database, the only integral PTS, fructose-specific PTS (PTS ${ }^{\mathrm{Fru}}$ ), was found in the genome of $R$. sphaeroides ATCC 17023, which is encoded by the gene cluster fruAB (RSP_1788 and RSP_1786). fruB encodes EI and HPr, the two sugar-nonspecific protein constituents of the PTS, and fruA encodes the sugarspecific transporter. It was reported that PTS ${ }^{\text {Fru }}$ encoded by fruAB simultaneously had a function of glucose transmembrane in some $E$. coil strains [6]. Additionally, fruAB in $R$. sphaeroides may have a similar function as that of the abovementioned E. coil strains. Glucose transported into cells with non-PTS must be phosphorylated before subsequent metabolism. Although the non-PTS-type glucose-specific transporter in $R$. sphaeroides ATCC 17023 has not been identified, the enzyme glucokinase ( $g l k$, RSP_2875), which plays a role in glucose phosphorylation, exists in the genome $[5,8]$. Additionally, the glucokinase activity had been determined in some $R$. sphaeroides strains when cultured with glucose as the sole carbon source [21]. Considering the above information, R. sphaeroides ATCC 17023 should possess the
non-PTS. R. sphaeroides ATCC 17023 metabolizes glucose exclusively with the Entner-Doudoroff pathway (ED) under aerobic and anaerobic conditions because of the lack of phosphofructokinase in the Embden-Meyerhof-Parnas pathway (EMP) [18]. According to the abovementioned analysis, metabolic networks that contain the glucose transmembrane and catabolism were constructed and the result is depicted in Fig. 1.

\section{Influence of PTS and non-PTS on bacterial growth and glucose metabolism}

To clarify whether PTS (fruAB), non-PTS, or both of $R$. sphaeroides ATCC 17023 influences cellular glucose metabolism, two mutants $(\triangle$ fruA $\Delta$ fruB and $\Delta g l k)$ were constructed using an in-frame markerless deletion method. The $\triangle$ fruA $\triangle$ fruB was a mutant with double knock out of fruA and fruB. Considering that no non-PTS type glucose transporter has been identified in $R$. sphaeroides presently, glk was knocked out to study the function of non-PTS in glucose metabolism. Subsequently, these mutants' growth and glucose consumption were studied under aerobic incubation using glucose as the sole carbon source (Fig. 2). All mutant strains showed a lag phase at the beginning of cultivation (between 0 and $12 \mathrm{~h}$ ), which was similar to that of the R. sphaeroides ATCC 17023 (WT) (Fig. 2a). Evident variations were observed among these strains since then. The WT went into an exponential growth phase and displayed a rapid

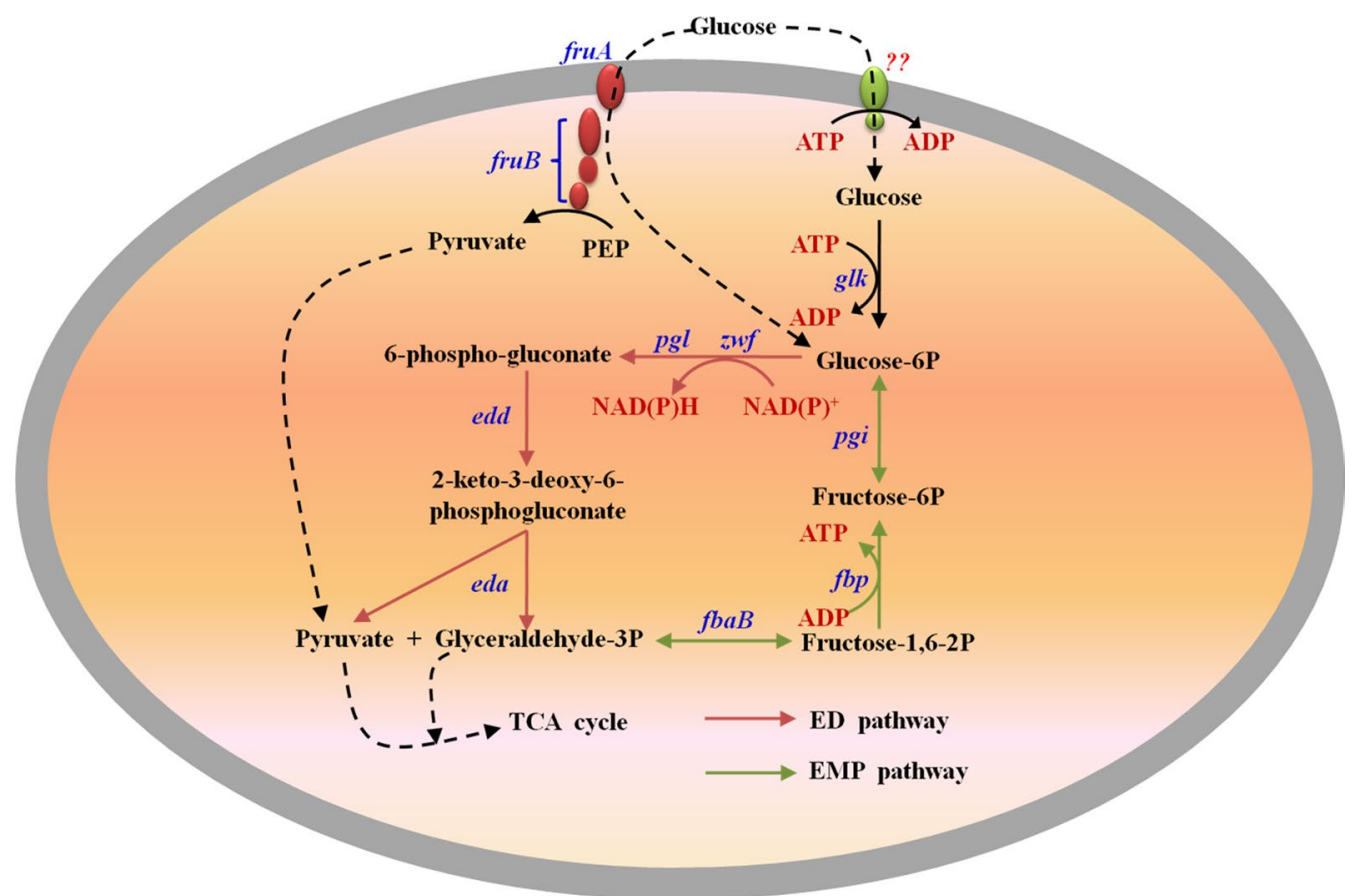

Fig. 1 Map of potential glucose transmembrane and metabolism pathways in R. sphaeroides ATCC 17023 


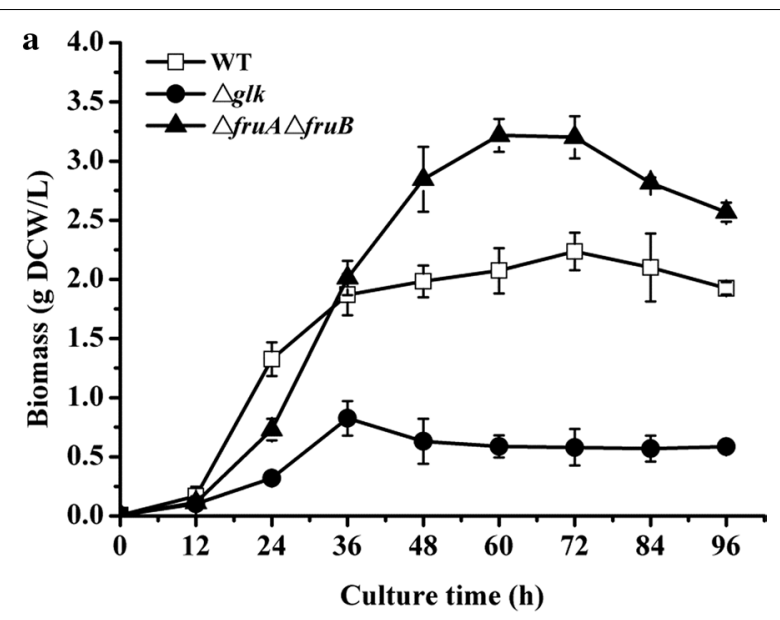

b

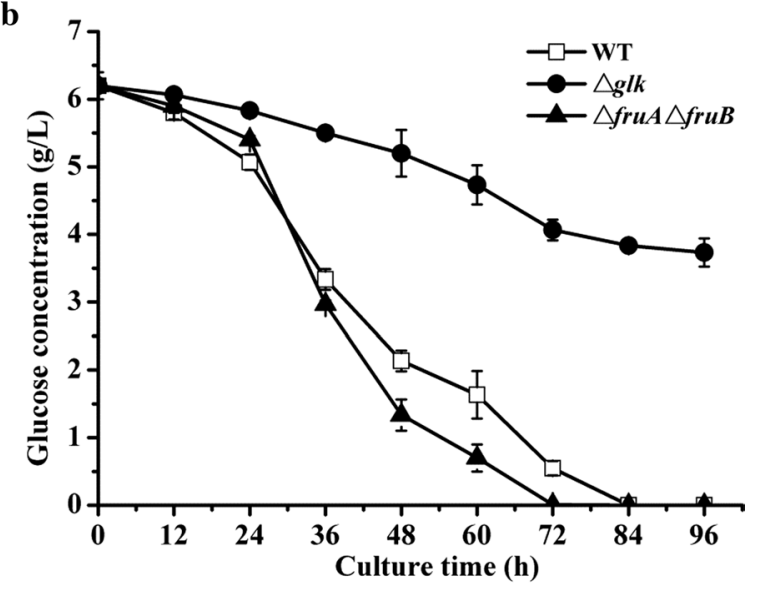

c

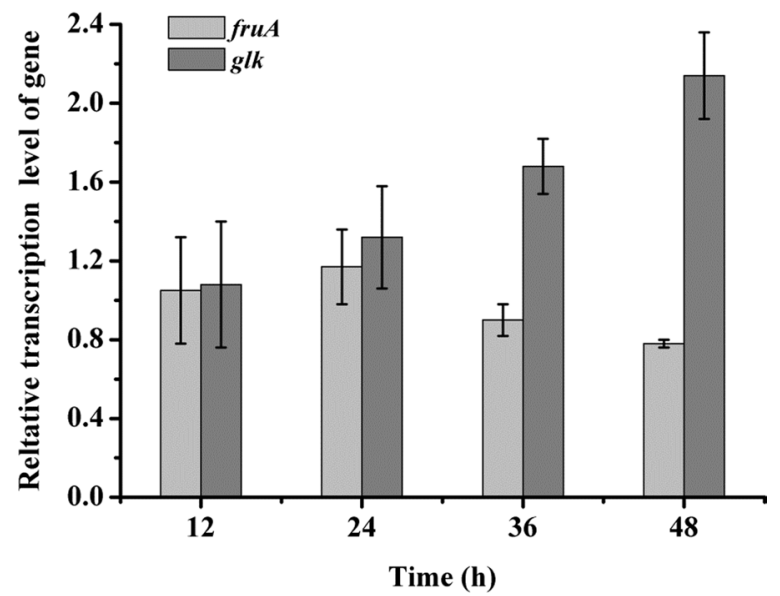

Fig. 2 Growth and glucose metabolism of the WT and the mutant strains cultured in the MSMM, and RT-qPCR assay of the fruA and $g / k$ in the WT at different culture time. a Growth curves, b glucose concentration curves, and $\mathbf{c}$ relative transcription level growth rate than others between 12 and $24 \mathrm{~h}$. Furthermore, the $\triangle$ fruA $\triangle$ fruB went into an exponential growth phase though the growth rate was slower than the WT; however, $\Delta g l k$ still showed a slow growth rate. After that, the growth rate of the WT became slower from 36 to $72 \mathrm{~h}$, going into a decline phase at $72 \mathrm{~h} . \Delta$ fruA $\Delta$ fruB showed a faster growth rate between 24 and $36 \mathrm{~h}$, and then the rate gradually slowed. The stationary growth phase was observed at approximately $60 \mathrm{~h}$, and the decline phase appeared at $72 \mathrm{~h}$ for $\triangle$ fruA $\Delta$ fruB. In contrast, $\Delta g l k$ continually kept a slow growth status between 24 and $36 \mathrm{~h}$ and went into a long stationary growth phase till the end of the experiment. Interestingly, the Biomax obtained by $\triangle$ fruA $\Delta$ fruB was $3.22 \pm 0.04 \mathrm{~g} D C W / \mathrm{L}$, which was much higher than that of the WT $\left(B_{\text {max }}\right.$ was $2.23 \pm 0.07 \mathrm{~g} \mathrm{DCW} / \mathrm{L}$ ) (Table 2). Although the $\Delta g l k$ showed a typical bacterial growth process, both the growth rate and $\mathrm{Bio}_{\max }$ were much weaker than the WT during the whole culture process that the $B i o_{\max }$ was $0.82 \pm 0.01 \mathrm{~g} \mathrm{DCW} / \mathrm{L}$ achieved by the $\Delta g l k$.

Additionally, glucose concentration was determined simultaneously (Fig. 2b). At the beginning of cultivation $(0-12 \mathrm{~h})$, the strains showed slow glucose consumption rates $\left(r_{g l c}\right)$ that fit the characteristics of the lag phase. During the culture time between 12 and $24 \mathrm{~h}$, the WT and $\triangle$ fruA $\triangle$ fruB sped up glucose consumption, though $\triangle$ fruA $\triangle$ fruB had a little slower consumption rate than the WT. The result could explain the reason why $\triangle$ fruA $\triangle$ fruB grew slower than the WT. Afterward, the residual glucose concentration in the group with $\triangle f r u A \Delta f r u B$ was less than the WT. Finally, $\triangle$ fruA $\triangle$ fruB exhausted the glucose within $72 \mathrm{~h}$, which was $12 \mathrm{~h}$ earlier than the WT. Compared with the WT, $\Delta g l k$ showed a low ability on glucose consumption during the entire process. After incubation for $96 \mathrm{~h}$, there was still $3.73 \pm 0.21 \mathrm{~g} / \mathrm{L}$ residual glucose in the medium. The $r_{g l c}$ of $\Delta g l k$ was only $0.026 \pm 0.001 \mathrm{~g} / \mathrm{L} / \mathrm{h}$, whereas the $r_{g l c}$ of the $\Delta$ fruA $\Delta$ fruB could get to $0.086 \pm 0.002 \mathrm{~g} / \mathrm{L} / \mathrm{h}$, which was approximately 1.18 times that of the WT (Table 2). Besides the $r_{g l c}$, the $Y_{b / g l c}$ of the $\triangle f r u A \Delta f r u B$ was also promoted, approximately $43.4 \%$ higher than that of WT. Additionally, we constructed the mutant strains, $\triangle f r u A$ and $\triangle$ fruB. The results revealed that the two mutants showed similar growth and glucose metabolism status as those of the $\triangle f r u A \Delta f r u B$ (unpublished data). Summarily, $g l k$ mutation seriously inhibited the growth and glucose metabolism of $R$. sphaeroides. Considering that $g l k$ is a vital gene involved in non-PTS, we speculated that the non-PTS played a major role in transporting glucose for R. sphaeroides ATCC 17023 during the entire process. 
Table 2 Growth and glucose consumption assay of the R. sphaeroides mutant strains and WT

\begin{tabular}{llllll}
\hline Strain & Time $(\mathbf{h})$ & $\begin{array}{l}\text { Glucose metabolism } \\
(\mathbf{g} / \mathbf{L})\end{array}$ & $\mathbf{B i o}_{\max }{ }^{*}(\mathbf{g ~ D C W} / \mathbf{L})$ & $\boldsymbol{r}_{\text {glc }^{* * *}}(\mathbf{g} / \mathbf{L} / \mathbf{h})$ & $\boldsymbol{Y}_{\text {b/glc }}{ }^{* * *}(\mathbf{g} / \mathbf{g})$ \\
\hline WT & $84^{\mathrm{b}}$ & $6.21 \pm 0.21^{\mathrm{a}}$ & $2.23 \pm 0.07^{\mathrm{b}}(72 \mathrm{~h})$ & $0.074 \pm 0.003^{\mathrm{b}}$ & $0.36 \pm 0.02^{\mathrm{b}}$ \\
$\Delta$ glk & $96^{\mathrm{a}}$ & $2.47 \pm 0.04^{\mathrm{b}}$ & $0.82 \pm 0.01^{\mathrm{c}}(96 \mathrm{~h})$ & $0.026 \pm 0.001^{\mathrm{c}}$ & $0.33 \pm 0.01^{\mathrm{c}}$ \\
$\triangle$ fruA $\triangle$ fruB & $72^{\mathrm{c}}$ & $6.19 \pm 0.16^{\mathrm{a}}$ & $3.22 \pm 0.04^{\mathrm{a}}(60 \mathrm{~h})$ & $0.086 \pm 0.002^{\mathrm{a}}$ & $0.52 \pm 0.03^{\mathrm{a}}$ \\
\hline
\end{tabular}

${ }^{*}$ Bio $_{\text {max }}$ the maximum biomass; ${ }^{* *} r_{g / c}$ the average glucose consumption rate; ${ }^{* * *} Y_{b / g l c}$ biomass yield to glucose consumption vs. the $B o_{\text {max }}$. Statistics analysis was performed based on one-way ANOVA method and the data in the same column with the same letters $(a-c)$ meant no significant difference $(P \leq 0.05)$

However, deleting fruAB also influenced bacterial growth and glucose metabolism. The depressing effect of the fru $A B$ mutation on growth appeared at the early incubation phase (12-24 h), whereas it showed a promotion effect on growth at the later phase (24-72 h). The influence of fruAB mutation on growth could be indirectly explained by the status of glucose metabolism. Therefore, we supposed that both the PTS and non-PTS had influence on glucose metabolism at the early culture phase. The relative transcriptional level of the fruA and $g l k$ in WT during cultivation was determined by RT-qPCR to verify the hypothesis further. The result was depicted in Fig. 2c, both fruA and $g l k$ showed an increasing tendency in the early culture phase (12-24 h). After that, the transcriptional level of fruA showed a decreased tendency since $36 \mathrm{~h}$, whereas the $g l k$ still kept increasing from 36 to $48 \mathrm{~h}$. Additionally, $\triangle$ fruA $\Delta$ fruB $\Delta g l k$ was obtained through deleting the gene $g l k$ on the basis of $\Delta f r u A \Delta f r u B$. Comparing with $\Delta g l k$, both growth and glucose utilization were inhibited further at the early culture phase (Additional file 4: Fig. S4). To sum up, the results illustrate that both non-PTS and PTS have a function on glucose metabolism during the early phase, and the nonPTS played an important role in glucose metabolism.

\section{Enhancing the non-PTS pathway to promote cellular glucose metabolism}

According to the above study, blocking the non-PTS inhibited the glucose metabolism of $R$. sphaeroides ATCC 17023. Whether over-expressing the non-PTS-type glucose transporter helps in improving glucose catabolism. In this section, the galactose: $\mathrm{H}^{+}$symporter ( galP) from $E$. coil $\mathrm{K}-12$ substr. MG1655 was selected for the study. First, three mutants, $\triangle$ fruA $\triangle$ fruB $/ \mathrm{bp}, \quad \Delta$ fruA $\triangle$ fruB $/$ galP $O P$, and $\triangle$ fruA $\triangle$ fruB/tac:: $g a l P_{O P}$, were constructed with the over-expression vectors, $\mathrm{pBBR} 1 \mathrm{MCS}-2$ and $\mathrm{pBBR} 1 \mathrm{MCS}$ $2:: t a c$. The $\triangle$ fruA $\triangle$ fruB/bp was directly introduced to the blank plasmid in $\triangle$ fruA $\triangle$ fruB. The $\triangle$ fruA $\triangle$ fruB/galP $O P$ was introduced to the plasmid, only harboring the gene, galP. The $\triangle$ fruA $\triangle$ fruB/tac::galP $P_{O P}$ is inserted with a strong promoter tac before the gene galP based on the pBBR1MCS-2::tac. Subsequently, these mutant strains were separately cultivated with glucose as the

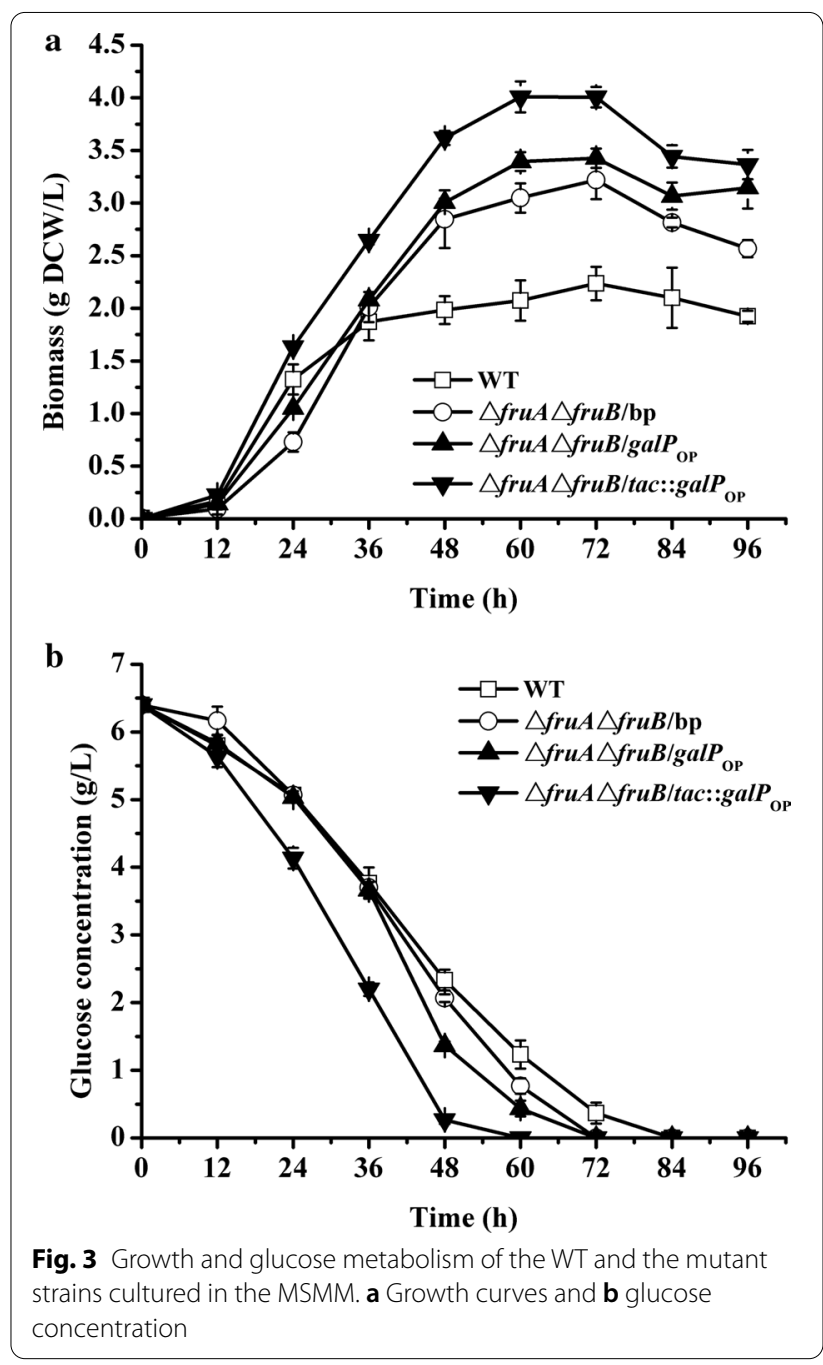

carbon source, and the biomass and glucose concentration was determined every $12 \mathrm{~h}$. The $\Delta$ fruA $\Delta$ fruB/bp showed almost no difference from that of $\triangle$ fruA $\triangle$ fruB in growth and glucose metabolism (Fig. 3). This means that the plasmid introduction did not influence bacterial growth and glucose metabolism. Compared with $\triangle$ fruA $\triangle$ fruB $/ \mathrm{bp}$, the growth rate of $\triangle$ fruA $\Delta$ fruB $/$ galP $_{O P}$ was increased at the early phase $(12-24 \mathrm{~h})$, but the 
Table 4 The $\mathrm{CoQ}_{10}$ content of WT and mutants cultured in SMM

\begin{tabular}{|c|c|c|c|c|}
\hline \multirow[t]{2}{*}{ Culture time (h) } & \multicolumn{4}{|c|}{$\mathrm{CoQ}_{10}$ content (mg/g DCW) } \\
\hline & WT & $\Delta g l k$ & $\Delta$ fruA $\Delta$ fruB & $\Delta$ fruA $\Delta$ fruB/tac:: galP ${ }_{O P}$ \\
\hline 24 & $3.79 \pm 0.11^{\mathrm{a}}$ & $3.12 \pm 0.04^{d}$ & $3.23 \pm 0.17^{c}$ & $3.43 \pm 0.16^{b}$ \\
\hline 48 & $3.62 \pm 0.06^{b}$ & $2.53 \pm 0.33^{c}$ & $3.65 \pm 0.05^{b}$ & $3.76 \pm 0.21^{\mathrm{a}}$ \\
\hline 72 & $3.83 \pm 0.13^{b}$ & $2.85 \pm 0.05^{c}$ & $4.97 \pm 0.15^{\mathrm{a}}$ & $5.01 \pm 0.33^{\mathrm{a}}$ \\
\hline 96 & $3.95 \pm 0.21^{c}$ & $3.02 \pm 0.19^{d}$ & $5.02 \pm 0.18^{b}$ & $5.11 \pm 0.14^{\mathrm{a}}$ \\
\hline
\end{tabular}

Statistical analysis was performed based on one-way ANOVA and the data with the same letters (a-e) means no significant difference $(\mathrm{P} \leq 0.05)$ for each line

growth status was the same as that of $\triangle f r u A \Delta f r u B / b p$ between 24 and $48 \mathrm{~h}$ (Fig. 3a). From $48 \mathrm{~h}$, the biomass achieved by $\triangle$ fruA $\triangle$ fruB/galP $O P$ was higher than that of $\triangle$ fru $A \triangle$ fruB/bp though the growth trends were similar. The higher biomass achieved by $\triangle$ fruA $\triangle$ fruB $/$ galP $_{O P}$ could be explained by the faster glucose consumption rate than the $\triangle f r u A \triangle f r u B /$ bp during this period. The result also suggested that the over-expression of galP could improve cellular glucose metabolism. For $\triangle$ fruA $\triangle$ fruB/tac:: galP $_{O P}$, the growth improved further than $\Delta$ fruA $\Delta$ fruB/galP $O P$ at the early phase $(12-24 \mathrm{~h})$, and then, it still kept a fast growth status than others until the time glucose was nearly exhausted. Additionally, the biomass quantity achieved was higher than that of $\triangle$ fruA $\triangle$ fruB/galP ${ }_{O P}$. The $B i o_{\max }$ was $4.01 \pm 0.15 \mathrm{~g}$ $\mathrm{DCW} / \mathrm{L}$ achieved by $\Delta$ fruA $\triangle$ fruB/tac::galP ${ }_{O P}$, which was the highest value among these strains. For glucose metabolism, $\Delta$ fruA $\Delta$ fruB/tac:: galP $_{O P}$ exhausted the glucose in the medium within $60 \mathrm{~h}$, and the $r_{g l c}$ reached $0.107 \pm 0.003 \mathrm{~g} / \mathrm{L} / \mathrm{h}$ (Table 3). Furthermore, glk was overexpressed in $\triangle$ fruA $\triangle$ fruB $(\triangle$ fruA $\Delta$ fruB/tac::glk). However, both the growth and glucose metabolism decreased compared with $\triangle$ fruA $\triangle$ fruB/bp (Additional file 5: Fig. S5). Additionally, the result suggested that the original $g l k$ transcriptional level was fitting for glucose metabolism. Maybe, over-expression of the $g l k$ produced excessive glucose-6-P, which is toxic to cells.

\section{Improving $\mathrm{CoQ}_{10}$ productivity of $R$. sphaeroides}

The $\mathrm{CoQ}_{10}$ content of these mutants was determined, and the result is presented in Table 4. Compared with the WT, $\Delta g l k, \Delta f r u A \Delta f r u B$, and $\Delta$ fruA $\Delta$ fruB/tac::galP $O P$ synthesized a low content of $\mathrm{CoQ}_{10}$ when incubated for $24 \mathrm{~h}$; especially, the $\mathrm{CoQ}_{10}$ content of $\Delta g l k$ was $3.12 \pm 0.04 \mathrm{mg} / \mathrm{g}$ DCW. After that, $\mathrm{CoQ}_{10}$ content of $\triangle$ fruA $\triangle$ fruB and $\triangle$ fruA $\Delta$ fruB/tac::galP $P_{O P}$ was increased after $48 \mathrm{~h}$, whereas the $\mathrm{CoQ}_{10}$ content of the WT and $\Delta g l k$ showed a slight reduction. As incubation proceeded (48-96 h), the $\mathrm{CoQ}_{10}$ content of the WT and $\Delta g l k$ stopped reducing and increased. Simultaneously, $\triangle$ fruA $\triangle$ fruB and $\triangle$ fruA $\triangle$ fruB/tac::galP $P_{O P}$ increased in the $\mathrm{CoQ}_{10}$ content. Finally, the $\mathrm{CoQ}_{10}$ content of $\triangle$ fruA $\Delta$ fruB and $\Delta$ fruA $\Delta$ fruB/tac:: $g a l P_{O P}$ reached $5.02 \pm 0.18$ and $5.11 \pm 0.14 \mathrm{mg} / \mathrm{g} \mathrm{DCW}$, respectively. The maximum $\mathrm{CoQ}_{10}$ content of $\triangle$ fruA $\triangle$ fruB/tac:: galP $_{O P}$ was increased by $29.4 \%$ than the WT. It can be proposed that the mutation of fruAB improved $Y_{b / g l c}$ and bacterial $r_{g l c}$ but also enhanced the $\mathrm{CoQ}_{10}$ synthesis of $R$. sphaeroides. Moreover, strengthening glucose transportation by overexpressing galP showed little help to strengthen $\mathrm{CoQ}_{10}$ synthesis.

Although galP over-expression in $\triangle$ fruA $\triangle$ fruB played no role in promoting the $C_{0} Q_{10}$ synthesis ability of $R$. sphaeroides, the strategy can promote glucose metabolism rate, which shortens the fermentation time. The inactivation of fruAB improved $Y_{b / g l c}$ and the bacterial $\mathrm{CoQ}_{10}$ synthetic ability. Considering the advantages of the two strategies, $\triangle$ fruA $\triangle$ fruB/tac::galP ${ }_{O P}$ was applied to $\mathrm{CoQ}_{10}$ fermentation in a lab-scale tank $(1 \mathrm{~L})$, evaluating whether the $\mathrm{CoQ}_{10}$ fermentation is improved. $\triangle$ fruA $\triangle$ fruB/tac:: $g a l P_{O P}$ showed an evident improvement in growth compared with the WT/bp during the

Table 3 Growth and glucose metabolism of R. sphaeroides strains

\begin{tabular}{|c|c|c|c|c|c|}
\hline Strain & Time (h) & $\begin{array}{l}\text { Glucose metabolism } \\
(\mathrm{g} / \mathrm{L})\end{array}$ & $\mathrm{Bio}_{\max }{ }^{*}(\mathrm{~g} \mathrm{DCW} / \mathrm{L})$ & $r_{g l c}^{* *}(g / L / h)$ & $Y_{\text {bio/glc }}^{* * *}(\mathrm{~g} / \mathrm{g})$ \\
\hline WT & $84^{\mathrm{b}}$ & $6.21 \pm 0.21^{\mathrm{a}}$ & $2.23 \pm 0.07^{d}(72 h)$ & $0.074 \pm 0.003^{c}$ & $0.36 \pm 0.02^{b}$ \\
\hline$\triangle$ fruA $\triangle$ fruB/bp & $72^{c}$ & $6.18 \pm 0.32^{\mathrm{a}}$ & $3.21 \pm 0.11^{c}(72 \mathrm{~h})$ & $0.086 \pm 0.002^{b}$ & $0.52 \pm 0.02^{c}$ \\
\hline$\triangle$ fruA $\triangle$ fruB/ galP $O P$ & $72^{c}$ & $6.21 \pm 0.12^{\mathrm{a}}$ & $3.43 \pm 0.17^{b}(72 h)$ & $0.086 \pm 0.008^{b}$ & $0.55 \pm 0.05^{b}$ \\
\hline$\triangle$ fruA $\triangle$ fruB/tac::galP $\mathrm{OP}$ & $60^{a}$ & $6.20 \pm 0.17^{\mathrm{a}}$ & $4.01 \pm 0.15^{\mathrm{a}}(60 \mathrm{~h})$ & $0.103 \pm 0.003^{\mathrm{a}}$ & $0.65 \pm 0.07^{\mathrm{a}}$ \\
\hline
\end{tabular}

${ }^{*}$ Bio $_{\text {max }}$ the maximum biomass; ${ }^{* *} r_{g l c}$ the average glucose consumption rate; ${ }^{* * *} Y_{\text {bio/glc }}$ biomass yield to glucose consumption versus the $B i o_{\text {max }}$. Statistics analysis was performed based on one-way ANOVA method and the data in the same column with the same letters (a-d) meant no significant difference ( $\mathrm{a} \leq 0.05)$ 

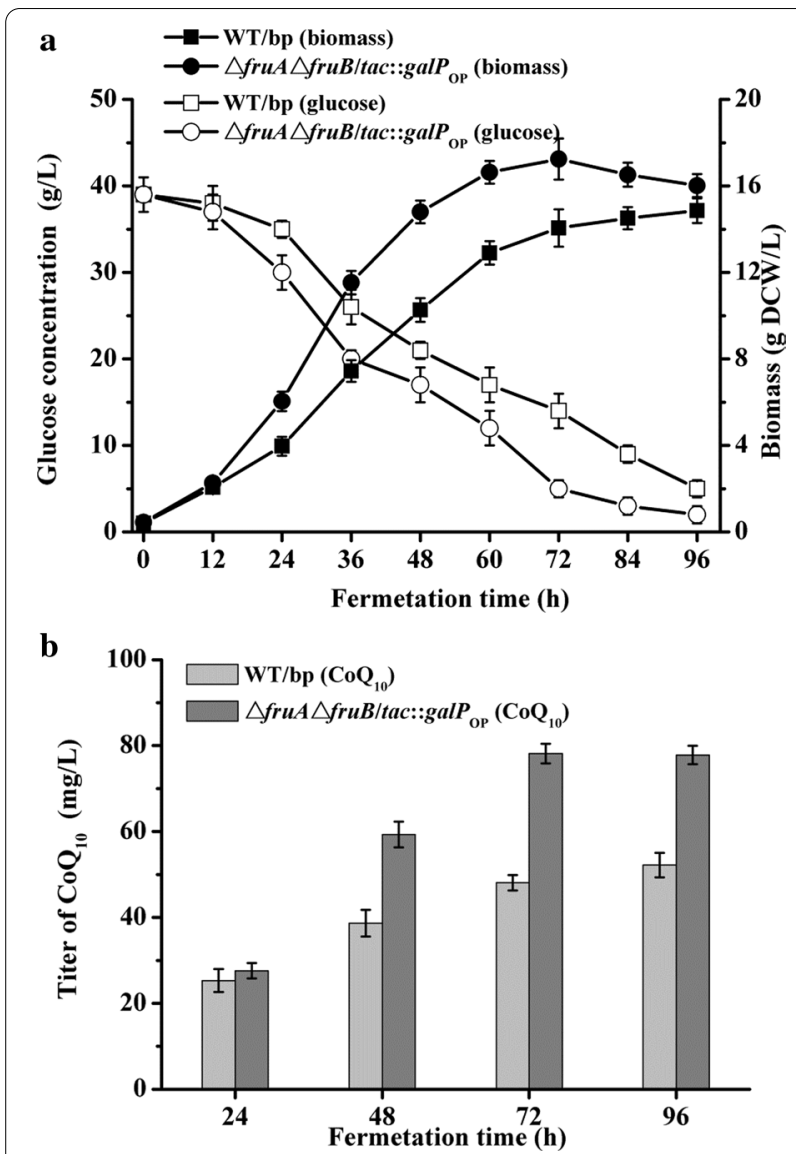

Fig. 4 Comparison of the growth, glucose metabolism and titer of $\mathrm{CoQ}_{10}$ of the WT and the $\triangle$ fruA $\triangle$ fruB/tac::galP ${ }_{O P}$ cultured in the fermentation medium B. a Growth and glucose concentration curves, and $\mathbf{b}$ Titer of $\mathrm{CoQ}_{10}$ during fermentation

fermentation process $\left(12-72 \mathrm{~h}\right.$ ) (Fig. 4a). The $\mathrm{Bio}_{\max }$ of $\triangle$ fruA $\Delta$ fruB/tac::galP $O P$ was harvested at $72 \mathrm{~h}$ of fermentation, which was $24 \mathrm{~h}$ earlier than the WT/bp. Moreover, the value of the $B i o_{\max }$ reached 17.24 $\pm 0.97 \mathrm{~g} \mathrm{DCW} / \mathrm{L}$, which was promoted by approximately $16 \%$ higher than that of the WT/bp $(14.85 \pm 0.57 \mathrm{~g} \mathrm{DCW} / \mathrm{L})$. Simultaneously, the glucose concentration in the medium was almost exhausted after $72 \mathrm{~h}$ for $\triangle$ fruA $\triangle$ fruB/tac:: galP $_{O P}$ $(<5 \mathrm{~g} / \mathrm{L})$, whereas there was more than 10 -g/L residual glucose residual for the WT/bp. In the aspect of $\mathrm{CoQ}_{10}$ synthesis (Fig. 4b), the yield gradually increased as the fermentation proceeded for both strains. At $48 \mathrm{~h}$ incubation, the yield of $\triangle$ fruA $\Delta$ fruB/tac::galP $O P$ showed a higher level than that of the WT/bp, and the phenomenon lasted to the end. The maximum $\mathrm{CoQ}_{10}$ titer of $\triangle$ fruA $\triangle$ fruB/tac:: galP $_{O P}$ reached $78.14 \pm 2.31 \mathrm{mg} / \mathrm{L}$, which was approximately $49.76 \%$ higher than that of the $\mathrm{WT} / \mathrm{bp}$. Moreover, $\triangle$ fruA $\triangle$ fruB/tac::galP ${ }_{O P}$ achieved the maximum $\mathrm{CoQ}_{10}$ titer at $72 \mathrm{~h}$, which was $24 \mathrm{~h}$ earlier than the WT/bp.

\section{Discussion}

For many bacteria, PTS ${ }^{\mathrm{Glc}}$ is the first-selected pathway to transport exogenous glucose $[6,22]$. After that, some other sugar-specific-PTSs, such as the PTS ${ }^{\mathrm{Fru}}($ fruAB), have been identified with the same function as that of the PTS ${ }^{\text {Glc }}[6,23]$. In $R$. sphaeroides ATCC 17023, no $\mathrm{PTS}^{\mathrm{Glc}}$-encoding genes are identified presently, but it has a PTS ${ }^{\text {Fru }}$ encoding gene cluster $($ fru $A B)$. It is revealed that glucose metabolism and bacterial growth were influenced by the mutation of the fruAB in $R$. sphaeroides ATCC 17023 (Fig. 2). Interestingly, the result of fruAB mutation revealed two effects on bacterial growth during the whole culture process. It showed an inhibition effect at the early cultivation phase (12-24 h) and displayed a promoting effect at the late phase at $36 \mathrm{~h}$. Finally, the $B i_{\text {max }}$ received by $\triangle$ fruA $\triangle$ fruB was much higher than that of the WT. The glucose metabolism of $\triangle$ fruA $\triangle$ fruB also displayed a fit change characteristic vs. that of the growth change. Additionally, RT-qPCR assay revealed that the transcription level of the fruA in the WT kept a relatively high level at the early cultivation phase (12-24 h) and then decreased at $36 \mathrm{~h}$. With the comprehensive analysis of the results, we supposed that the PTS ${ }^{\mathrm{Fru}}$ in $R$. sphaeroides ATCC 17023 majorly joined in the glucose metabolism at the early cultivation phase. The PTS ${ }^{\mathrm{Glc}}$ is considered an effective way to utilize glucose because only one PEP is coupled with the translocation-phosphorylation of PTS carbohydrates when forming one ATP [6, 22]. In contrast, glucose utilization through a non-PTS transporter requires extra ATP to phosphate a carbohydrate molecule in the carbohydrate kinase reaction. Regarding energy consumption, bacteria synthesize cellular skeleton materials at the early cultivation phase, requiring a large amount of energy; thus, the PTS type system may be a good choice for saving energy at the early growth phase. As cultivation continued, the function of $\operatorname{fr} u A B$ was weakened. The result might be due to the energy production ability of the bacteria, which is not as a limiting factor for cell growth after the lag phase. The high biomass achieved by $\triangle$ fruA $\triangle$ fruB meant that more carbon fluxed to cellular assimilation metabolism. For the native glucose utilization pathway in E. coli, half of the PEP produced is used for glucose uptake and phosphorylation. PEP is an essential precursor for synthesizing many chemicals, such as succinate, malate, and aromatic compounds. In this sense, fruAB mutation might reduce PEP catabolism, which helps to synthesize cytoskeleton substances after the lag growth phase. The similar phenomenon is also found in the mutation of PTS ${ }^{\mathrm{Ntr}}$ in P. putida, which is due to the enhancement of anabolism [16]. The phenomenon obtained from fruAB mutation is interesting though the mechanism is unclear. In future studies, 
more efforts will be put on disclosing the mechanism for promoting growth and its use.

The non-PTS composes of sugar transporters and glucokinase $(g l k)$. Glucose transports into cells by nonPTS in a non-phosphorylated form and then phosphorylated by the glucokinase for subsequent metabolism. A $g l k$ gene exists in the genome of $R$. sphaeroides ATCC 17023. The result revealed that the mutation of $g l k$ decreased bacterial growth when cultured in the medium with glucose as the sole carbon source. In addition, a poor glucose consumption status was observed for the mutant $\Delta g l k$. RT-qPCR assay revealed that the transcriptional level of $g l k$ in WT kept an increased tendency as the cultivation continued. The above results revealed that the non-PTS played an important role in controlling glucose metabolism of R. sphaeroides ATCC 17023 during the culture process, especially since exponential growth phase, though the corresponding sugar-specific transporters were still unidentified. The result agrees with other $R$. sphaeroides strains [18].

Galactose permease (galP) is a galactose: $\mathrm{H}^{+}$symporter belonging to the MFS [8]. It was reported that the $E$. coli $\mathrm{PTS}^{-}$glucose ${ }^{+}$strain could transport glucose by a non-PTS mechanism as fast as its WT parental strain [23]. Further research showed that the gal regulon genes, which encode non-PTS transporter and enzymes for galactose metabolism, are enhanced in this mutant; furthermore, rapid glucose consumption depends on the low-affinity GalP. However, the overexpression of a heterogeneous galP in $\triangle$ fruA $\triangle$ fruB improved the decrease in growth generated by the fru $A B$ mutation at the early cultivation phase (Fig. 3 ). Alternatively, it could enhance glucose metabolism and promote biomass accumulation during the entire cultivation process. The results further illustrated that $R$. sphaeroides ATCC 17023 glucose metabolism mainly relied on the non-PTS. Additionally, it suggested the glucose transmembrane was an important limitation for the glucose metabolism of this bacterium. Furthermore, over-expressing $g l k$ was harmful to bacterial growth (Additional file 5: Fig. S5); bacterial glucose metabolism was also inhibited. Excessive glucose-6-p might be accumulated in the cytoplasm because of the over-expression of $g l k$, which has toxic effect on bacterial metabolism. Finding an appropriate expression level of $g l k$ may solve the question.

Mutation of the fruAB influenced glucose metabolism and mediated the synthesis of $\mathrm{CoQ}_{10}$ in $R$. sphaeroides ATCC 17023. Presently, the mechanism of fruAB mutation on increasing bacterial $\mathrm{CoQ}_{10}$ synthesis is unknown. PEP is an important precursor for synthesizing aromatic compounds by the shikimate pathway. Aromatic compounds are vital sources of the benzene ring of $\mathrm{CoQ}_{10}$. Additionally, we previously knocked out the pyruvate kinase ( $p y k A)$ of $R$. sphaeroides ATCC 17023, transforming PEP to pyruvate. The mutant showed higher $\mathrm{CoQ}_{10}$ content than that of the WT (unpublished data). Considering the relationship between PEP and $C_{0} Q_{10}$, we supposed that PTS ${ }^{\mathrm{Fru}}$ inactivation might reduce the catabolism quantity of PEP, which was promoted more PEP flow to $\mathrm{CoQ}_{10}$ synthesis. However, the $\mathrm{CoQ}_{10}$ content of $\triangle$ fruA $\triangle$ fruB/tac::galP ${ }_{O P}$ showed no evident increase compared with that of $\triangle$ fruA $\triangle$ fruB. The result suggested that only enhancing glucose transport cannot promote bacterial $\mathrm{CoQ}_{10}$ synthesis ability.

\section{Conclusion}

The two sugar transmembrane pathways in $R$. sphaeroides ATCC 17023 influenced growth and glucose metabolism. The PTS $^{\text {Fru }}$ mutation revealed two effects on bacterial growth: inhibition at the early cultivation phase and promotion later. $g l k$ mutation decreased growth and glucose metabolism. Additionally, compared with the non-PTS, PTS ${ }^{\text {Fru }}$ had a relationship with $\mathrm{CoQ}_{10}$ synthesis that destroying the PTS $^{\text {Fru }}$ could enhance bacterial $\mathrm{CoQ}_{10}$ synthesis ability. Enhancing glucose transport of the non-PTS with over-expressing a galactose: $\mathrm{H}^{+}$symporter (galP) in $\triangle f r u A \Delta f r u B$ relieved the inhibition effect and enhanced growth. Moreover, the over-expression of galP has little effect on enhancing bacterial $\mathrm{CoQ}_{10}$ synthesis ability. According to the functional study of fruAB and $g l k$, $\mathrm{CoQ}_{10}$ fermentation was improved through several modifications in glucose metabolism (constructed the $\triangle$ fruA $\triangle$ fruB $/$ tac:: $\left.g a l P_{O P}\right)$ and was verified as available for fermentation in $1 \mathrm{~L}$ bioreactors. Summarily, our study provided a new guidance for improving $\operatorname{CoQ}_{10}$ productivity of $R$. sphaeroides.

\section{Supplementary Information}

The online version contains supplementary material available at https://doi. org/10.1186/s12934-021-01695-z.

Additional file 1: Fig. S1 (a) Construction flowchart of the glk gene deletion vector pK18mobsacB::glk-L-R; (b) Construction of glk gene deletion vector pK18mobsacB::g/k-L-R; and (c) Filtration and verification of $\Delta g / k$.

Additional file 2: Fig. S2 (a) Construction flowchart of the fruB gene deletion vector pK18mobsacB::fruB-L-R; (b) Construction of glk gene deletion vector pK18mobsacB:: fruA-L-R; (c) Construction of glk gene deletion vector pK18mobsacB:: fruB -L-R; (d) Filtration and verification of $\triangle$ fruA; and (e) Filtration and verification of $\triangle$ fruA $\triangle$ fruB.

Additional file 3: Fig. S3 (a) Construction flowchart of the galP gene overexpression vector pBBR1MCS-2::tac:;galP; (b) Construction of he galP 
gene overexpression vector pBBR1MCS-2::tac:;galP; and (c) Filtration and verification of the $\triangle$ fruA $\triangle$ fruB/tac:: galP $P_{O P}$.

Additional file 4: Fig. S4 Growth and glucose metabolism of the WT and the mutant strains cultured in the MSMM. (a) Growth curves and (b) glucose concentration curves.

Additional file 5: Fig. S5 Growth and glucose metabolism of the $\triangle$ fruA $\triangle$ fruB/bp and the $\triangle$ fruA $\triangle$ fruB/tac::glk cultured in the MSMM.

Additional file 6: Table S1 Primers and restriction enzymes used in this study.

Additional file 7: Table S2 Primers used for mutant strains verification.

Additional file 8: Table S3 Primers used for RT-qPCR amplification.

\section{Funding}

This study was funded by the Project was Supported by the Foundation (No. 202012) of Qilu University of Technology of Cultivating Subject for Biology and Biochemistry, National Natural Science Foundation of China (31800108), Natural Science Foundation of Shandong (ZR2019QC019), Key Technology Research and Development Program of Shandong (2019GHY112026), and Key Research and Development Plan of Shandong Province (2018YYSP018).

\section{Declarations}

Ethics approval and consent to participate

This article does not contain any studies with human participants or animals performed by any of the authors.

\section{Competing interests}

The authors declare that they have no conflict of interest.

Received: 11 September 2021 Accepted: 18 October 2021

Published online: 30 October 2021

\section{References}

1. Li F, Zhao Y, Li B, Qiao J, Zhao G. Engineering Escherichia coli for production of 4-hydroxymandelic acid using glucose-xylose mixture. Microb Cell Fact. 2016;15:90.

2. Tong $X$, Oh EK, Lee BH, Lee JK. Production of long-chain free fatty acids from metabolically engineered Rhodobacter sphaeroides heterologously producing periplasmic phospholipase A2 in dodecane-overlaid twophase culture. Microbial Cell Fact. 2019;18.

3. Wang S, Fang Y, Wang Z, Zhang S, Wang X. Improving L-threonine production in Escherichia coli by elimination of transporters ProP and ProVWX. Microb Cell Fact. 2021;20:58.

4. Wurm DJ, Veiter L, Ulonska S, Eggenreich B, Herwig C, Spadiut O. The E. coli pET expression system revisited-mechanistic correlation between glucose and lactose uptake. Appl Microbiol Biotechnol. 2016;100:8721-9.

5. Poblete-Castro I, Binger D, Rodrigues A, Becker J, Martins dos Santos VAP, Wittmann C. In-silico-driven metabolic engineering of Pseudomonas putida for enhanced production of poly-hydroxyalkanoates. Metabol Eng. 2013;15:113-23.
6. Luo Y, Zhang T, Wu H. The transport and mediation mechanisms of the common sugars in Escherichia coli. Biotechnol Adv. 2014;32:905-19.

7. Wurm DJ, Hausjell J, Ulonska S, Herwig C, Spadiut O. Mechanistic platform knowledge of concomitant sugar uptake in Escherichia coli BL21(DE3) strains. Sci Rep. 2017;7:45072.

8. Fordjour E, Adipah FK, Zhou S, Du G, Zhou J. Metabolic engineering of Escherichia coli BL21 (DE3) for de novo production of L-DOPA from D-glucose. Microb Cell Fact. 2019;18:74.

9. Chavarría M, Kleijn RJ, Sauer U, Pflüger-Grau K, Lorenzo VD. Regulatory tasks of the phosphoenolpyruvate-phosphotransferase system of Pseudomonas putida in central carbon metabolism. MBio. 2012;3:e00028-e112.

10. Lu J, Tang J, Liu Y, Zhu X, Zhang T, Zhang X. Combinatorial modulation of galP and glk gene expression for improved alternative glucose utilization. Appl Microbiol Biotechnol. 2012;93:2455-62.

11. Ikeda M. Sugar transport systems in Corynebacterium glutamicum: features and applications to strain development. Appl Microbiol Biotechnol. 2012;96:1191-200.

12. Gonzy-Tréboul G, Zagorec M, Rain-Guion MC, Steinmetz M. Phosphoenolpyruvate:sugar phosphotransferase system of Bacillus subtilis: nucleotide sequence of pts X, ptsH and the 5'-end of pts/ and evidence for a ptsHl operon. Mol Microbiol. 2010;3:103-12.

13. Su A, Chi S, Li Y, Tan S, Qiang S, Chen Z, Meng Y. Metabolic redesign of Rhodobacter sphaeroides for lycopene production. J Agric Food Chem. 2018;66:5879-85.

14. Zhang J, Gao D, Cai J, Liu H, Qi Z. Improving coenzyme $Q_{10}$ yield of Rhodobacter sphaeroides via modifying redox respiration chain. Biochem Eng J. 2018;135:98-104.

15. Chen X, Jiang X, Xu M, Zhang M, Huang R, Huang J, Qi F. Co-production of farnesol and coenzyme $Q_{10}$ from metabolically engineered Rhodobacter sphaeroides. Microb Cell Fact. 2019;18:98.

16. Lee $S$, Tan TS, Kawamukai M, Chen ES. Cellular factories for coenzyme $Q_{10}$ production. Microb Cell Fact. 2017;16:39.

17. Raizner $A E$, Quiones MA. Coenzyme $Q_{10}$ for patients with cardiovascular disease. J Am Coll Cardiol. 2021;77:609-19.

18. Imam S, Noguera DR, Donohue TJ. CceR and AkgR regulate central carbon and energy metabolism in Alphaproteobacteria. MBio. 2015;6:e02461-e12414.

19. Fuhrer T, Fischer E, Sauer U. Experimental identification and quantification of glucose metabolism in seven bacterial species. J Bacteriol. 2005; 187:1581-90.

20. Luo Y, Ge M, Wang B, Sun C, Wang J, Dong Y, Xi J. CRISPR/Cas9-deaminase enables robust base editing in Rhodobacter sphaeroides 2.4.1. Microbial Cell Fact. 2021;19:93.

21. Szymona M, Doudoroff M. Carbohydrate metabolism in Rhodopseudomonas sphreoides. J Gen Microbiol. 1960;22:167-83.

22. Kyselova L, Kreitmayer D, Kremling A, Bettenbrock K. Type and capacity of glucose transport influences succinate yield in two-stage cultivations. Microb Cell Fact. 2018;17:132.

23. Saier MH Jr. Families of transmembrane sugar transport proteins. Mol Microbiol. 2000;35:699-710.

\section{Publisher's Note}

Springer Nature remains neutral with regard to jurisdictional claims in published maps and institutional affiliations. 